\title{
Fractional trapezium type inequalities for twice differentiable preinvex functions and their applications
}

\author{
Artion Kashuri* and Rozana Liko \\ Department of Mathematics, Faculty of Technical Science, University Ismail Qemali, Vlora, Albania \\ artionkashuri@gmail.com,rozanaliko86@gmail.com
}

\section{ARTICLE INFO}

\section{Article History:}

Received 28 February 2019

Accepted 19 February 2020

Available 01 July 2020

\section{Keywords:}

Trapezium type integral inequalities

Preinvexity

General fractional integrals

AMS Classification 2010:

26A51; 26A33; 26D07; 26D10; 26D15

\section{ABSTRACT}

Trapezoidal inequalities for functions of divers natures are useful in numerical computations. The authors have proved an identity for a generalized integral operator via twice differentiable preinvex function. By applying the established identity, the generalized trapezoidal type integral inequalities have been discovered. It is pointed out that the results of this research provide integral inequalities for almost all fractional integrals discovered in recent past decades. Various special cases have been identified. Some applications of presented results to special means have been analyzed. The ideas and techniques of this paper may stimulate further research.

\section{$(c c) \mathrm{EY}$}

\section{Introduction}

The following inequality, named HermiteHadamard inequality, is one of the most famous inequalities in the literature for convex functions.

Theorem 1. Let $f: I \subseteq \mathbb{R} \longrightarrow \mathbb{R}$ be a convex function and $a_{1}, a_{2} \in I$ with $a_{1}<a_{2}$. Then the following inequality holds:

$$
\begin{gathered}
f\left(\frac{a_{1}+a_{2}}{2}\right) \leq \frac{1}{a_{2}-a_{1}} \int_{a_{1}}^{a_{2}} f(x) d x \\
\leq \frac{f\left(a_{1}\right)+f\left(a_{2}\right)}{2} .
\end{gathered}
$$

This inequality (1) is also known as trapezium inequality.

The trapezium inequality has remained an area of great interest due to its wide applications in the field of mathematical analysis. Authors of recent decades have studied (11) in the premises of newly invented definitions due to motivation of convex function. Interested readers see the references [1[16, [19, 20, 22, 23.

The aim of this paper is to establish trapezoidal type generalized integral inequalities for preinvex functions. Interestingly, the special cases of presented results, are fractional integral inequalities. Therefore, it is important to summarize the study of fractional integrals. Let us recall some special functions and evoke some basic definitions as follows:

Definition 1. [13] Let $f \in L\left[a_{1}, a_{2}\right]$. Then $k^{-}$ fractional integrals of order $\alpha, k>0$ with $a_{1} \geq 0$ are defined by

$I_{a_{1}^{+}}^{\alpha, k} f(x)=\frac{1}{k \Gamma_{k}(\alpha)} \int_{a_{1}}^{x}(x-t)^{\frac{\alpha}{k}-1} f(t) d t, \quad x>a_{1}$

and

$I_{a_{2}^{-}}^{\alpha, k} f(x)=\frac{1}{k \Gamma_{k}(\alpha)} \int_{x}^{a_{2}}(t-x)^{\frac{\alpha}{k}-1} f(t) d t, \quad a_{2}>x$.

For $k=1, k$-fractional integrals give RiemannLiouville integrals. For $\alpha=k=1, k$-fractional integrals give classical integrals.

Definition 2. [21] $A$ set $S \subseteq \mathbb{R}^{n}$ is said to be invex set with respect to the mapping $\eta: S \times S \longrightarrow$ $\mathbb{R}^{n}$, if $x+\operatorname{t\eta }(y, x) \in S$ for every $x, y \in S$ and $t \in[0,1]$.

The invex set also termed as, an $\eta$-connected set.

*Corresponding Author 
Definition 3. Let $S \subseteq \mathbb{R}^{n}$ be an invex set with respect to $\eta: S \times S \longrightarrow \mathbb{R}^{n}$. A function $f: S \longrightarrow[0,+\infty)$ is said to be preinvex with respect to $\eta$, if for every $x, y \in S$ and $t \in[0,1]$,

$$
f(x+t \eta(y, x)) \leq(1-t) f(x)+t f(y) .
$$

Also, let define a function $\varphi:[0,+\infty) \longrightarrow$ $[0,+\infty)$ satisfying the following conditions:

$$
\begin{gathered}
\int_{0}^{1} \frac{\varphi(t)}{t} d t<+\infty \\
\frac{1}{A} \leq \frac{\varphi(s)}{\varphi(r)} \leq A \text { for } \frac{1}{2} \leq \frac{s}{r} \leq 2 \\
\frac{\varphi(r)}{r^{2}} \leq B \frac{\varphi(s)}{s^{2}} \text { for } s \leq r \\
\left|\frac{\varphi(r)}{r^{2}}-\frac{\varphi(s)}{s^{2}}\right| \leq C|r-s| \frac{\varphi(r)}{r^{2}} \text { for } \frac{1}{2} \leq \frac{s}{r} \leq 2,
\end{gathered}
$$

where $A, B, C>0$ are independent of $r, s>0$. If $\varphi(r) r^{\alpha}$ is increasing for some $\alpha \geq 0$ and $\frac{\varphi(r)}{r^{\beta}}$ is decreasing for some $\beta \geq 0$, then $\varphi$ satisfies (3) $-(\underline{6})$, see [18]. Therefore, the left-sided and right-sided generalized integral operators are defined as follows:

$$
\begin{aligned}
& a_{1}^{+} I_{\varphi} f(x)=\int_{a_{1}}^{x} \frac{\varphi(x-t)}{x-t} f(t) d t, \quad x>a_{1}, \\
& a_{2}^{-} I_{\varphi} f(x)=\int_{x}^{a_{2}} \frac{\varphi(t-x)}{t-x} f(t) d t, \quad x<a_{2} .
\end{aligned}
$$

The most important feature of generalized integrals is that; they produce Riemann-Liouville fractional integrals, $k$-Riemann-Liouville fractional integrals, Katugampola fractional integrals, conformable fractional integrals, Hadamard fractional integrals, etc.

Motivated by the above literatures, the main objective of this paper is to discover in section 2, an interesting identity in order to study some new bounds regarding general trapezoidal type integral inequalities. By using the established identity as an auxiliary result, some new estimates for trapezoidal type integral inequalities via generalized integrals are obtained. It is pointed out that some new fractional integral inequalities have been deduced from main results. In section 3 some applications to special means are given. In section 4, a briefly conclusion is provided as well. The ideas and techniques of this paper may stimulate further research in the field of integral inequalities.

\section{Main results}

Throughout this study, let $P=\left[m a_{1}, a_{2}\right]$ with $a_{1}<a_{2}, m \in(0,1]$ be an invex subset with respect to $\eta: P \times P \longrightarrow \mathbb{R}$. Also, for brevity, we define

$$
\begin{gathered}
\Lambda_{m, n}^{(1)}(t)=\int_{0}^{t} \Delta_{m, n}^{(1)}(s) d s, \\
\Delta_{m, n}^{(1)}(s)=\int_{0}^{s} \frac{\varphi\left(\frac{\eta\left(x, m a_{1}\right)}{n+1} u\right)}{u} d u<+\infty,
\end{gathered}
$$

where $\eta\left(x, m a_{1}\right)>0$ and

$$
\begin{gathered}
\Lambda_{m, n}^{(2)}(t)=\int_{0}^{t} \Delta_{m, n}^{(2)}(s) d s, \\
\Delta_{m, n}^{(2)}(s)=\int_{0}^{s} \frac{\varphi\left(\frac{\eta\left(a_{2}, m x\right)}{n+1} u\right)}{u} d u<+\infty,
\end{gathered}
$$

where $\eta\left(a_{2}, m x\right)>0$.

For establishing some new results regarding general fractional integrals we need to prove the following lemma.

Lemma 1. Let $f: P \longrightarrow \mathbb{R}$ be a twice differentiable mapping on $\left(m a_{1}, a_{2}\right)$. If $f^{\prime \prime} \in L(P)$, then the following identity for generalized fractional integrals hold:

$$
\begin{aligned}
& \frac{\eta\left(x, m a_{1}\right) \Lambda_{m, n}^{(1)}(1)}{(n+1) \Delta_{m, n}^{(1)}(1)} \\
& \times \frac{f^{\prime}\left(m a_{1}\right)+f^{\prime}\left(m a_{1}+\eta\left(x, m a_{1}\right)\right)}{2} \\
& -\frac{f\left(m a_{1}\right)+f\left(m a_{1}+\eta\left(x, m a_{1}\right)\right)}{2}-\frac{1}{2 \Delta_{m, n}^{(1)}(1)} \\
& \times\left[\left(m a_{1}\right)^{+} I_{\varphi} f\left(m a_{1}+\frac{\eta\left(x, m a_{1}\right)}{n+1}\right)\right. \\
& \left.+{ }_{\left(m a_{1}+\eta\left(x, m a_{1}\right)\right)}-I_{\varphi} f\left(m a_{1}+\frac{n}{n+1} \eta\left(x, m a_{1}\right)\right)\right] \\
& +\frac{\eta\left(a_{2}, m x\right) \Lambda_{m, n}^{(2)}(1)}{(n+1) \Delta_{m, n}^{(2)}(1)} \\
& \times \frac{f^{\prime}(m x)+f^{\prime}\left(m x+\eta\left(a_{2}, m x\right)\right)}{2} \\
& -\frac{f(m x)+f\left(m x+\eta\left(a_{2}, m x\right)\right)}{2}-\frac{1}{2 \Delta_{m, n}^{(2)}(1)} \\
& \times\left[{ }_{(m x)^{+}} I_{\varphi} f\left(m x+\frac{\eta\left(a_{2}, m x\right)}{n+1}\right)\right. \\
& \left.+_{\left(m x+\eta\left(a_{2}, m x\right)\right)-} I_{\varphi} f\left(m x+\frac{n}{n+1} \eta\left(a_{2}, m x\right)\right)\right] \\
& =\frac{\eta^{2}\left(x, m a_{1}\right)}{2(n+1)^{2} \Delta_{m, n}^{(1)}(1)} \\
& \times \int_{0}^{1} \Lambda_{m, n}^{(1)}(t)\left[f^{\prime \prime}\left(m a_{1}+\frac{(n+t)}{n+1} \eta\left(x, m a_{1}\right)\right)\right. \\
& \left.-f^{\prime \prime}\left(m a_{1}+\frac{(1-t)}{n+1} \eta\left(x, m a_{1}\right)\right)\right] d t
\end{aligned}
$$

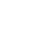




$$
\begin{gathered}
+\frac{\eta^{2}\left(a_{2}, m x\right)}{2(n+1)^{2} \Delta_{m, n}^{(2)}(1)} \\
\times \int_{0}^{1} \Lambda_{m, n}^{(2)}(t)\left[f^{\prime \prime}\left(m x+\frac{(n+t)}{n+1} \eta\left(a_{2}, m x\right)\right)\right. \\
\left.-f^{\prime \prime}\left(m x+\frac{(1-t)}{n+1} \eta\left(a_{2}, m x\right)\right)\right] d t
\end{gathered}
$$

We denote

$$
\begin{gathered}
I_{f, \Lambda_{m, n}^{(1)}, \Lambda_{m, n}^{(2)}, \Delta_{m, n}^{(1)}, \Delta_{m, n}^{(2)}}\left(x, a_{1}, a_{2}\right) \\
=\frac{\eta^{2}\left(x, m a_{1}\right)}{2(n+1)^{2} \Delta_{m, n}^{(1)}(1)} \\
\times \int_{0}^{1} \Lambda_{m, n}^{(1)}(t)\left[f^{\prime \prime}\left(m a_{1}+\frac{(n+t)}{n+1} \eta\left(x, m a_{1}\right)\right)\right. \\
\left.-f^{\prime \prime}\left(m a_{1}+\frac{(1-t)}{n+1} \eta\left(x, m a_{1}\right)\right)\right] d t \\
+\frac{\eta^{2}\left(a_{2}, m x\right)}{2(n+1)^{2} \Delta_{m, n}^{(2)}(1)} \\
\times \int_{0}^{1} \Lambda_{m, n}^{(2)}(t)\left[f^{\prime \prime}\left(m x+\frac{(n+t)}{n+1} \eta\left(a_{2}, m x\right)\right)\right. \\
\left.-f^{\prime \prime}\left(m x+\frac{(1-t)}{n+1} \eta\left(a_{2}, m x\right)\right)\right] d t .
\end{gathered}
$$

Proof. Integrating by parts twice (12) and changing the variables of integration, we have

$$
\begin{gathered}
I_{f, \Lambda_{m, n}^{(1)}, \Lambda_{m, n}^{(2)}, \Delta_{m, n}^{(1)}, \Delta_{m, n}^{(2)}}\left(x, a_{1}, a_{2}\right) \\
=\frac{\eta^{2}\left(x, m a_{1}\right)}{2(n+1)^{2} \Delta_{m, n}^{(1)}(1)} \\
\times\left\{\left.\frac{(n+1) \Lambda_{m, n}^{(1)}(t) f^{\prime}\left(m a_{1}+\frac{(n+t)}{n+1} \eta\left(x, m a_{1}\right)\right)}{\eta\left(x, m a_{1}\right)}\right|_{0} ^{1}\right. \\
\times \int_{0}^{1} \Delta_{m, n}^{(1)}(t) f^{\prime}\left(m a_{1}+\frac{(n+t)}{n+1} \eta\left(x, m a_{1}\right)\right) d t \\
+\left.\frac{(n+1) \Lambda_{m, n}^{(1)}(t) f^{\prime}\left(m a_{1}+\frac{(1-t)}{n+1} \eta\left(x, m a_{1}\right)\right)}{\eta\left(x, m a_{1}\right)}\right|_{0} ^{1} \\
\times \int_{0}^{1} \Delta_{m, n}^{(1)}(t) f^{\prime}\left(m a_{1}+\frac{(1-t)}{n+1} \eta\left(x, m a_{1}\right)\right) d t \\
+\frac{\eta^{2}\left(a_{2}, m x\right)}{2(n+1)^{2} \Delta_{m, n}^{(2)}(1)} \\
\times\left\{\left.\frac{(n+1) \Lambda_{m, n}^{(2)}(t) f^{\prime}\left(m x+\frac{(n+t)}{n+1} \eta\left(a_{2}, m x\right)\right)}{\eta\left(a_{2}, m x\right)}\right|_{0} ^{1}\right.
\end{gathered}
$$

$$
\begin{aligned}
& -\frac{(n+1)}{\eta\left(a_{2}, m x\right)} \\
& \times \int_{0}^{1} \Delta_{m, n}^{(2)}(t) f^{\prime}\left(m x+\frac{(n+t)}{n+1} \eta\left(a_{2}, m x\right)\right) d t \\
& +\left.\frac{(n+1) \Lambda_{m, n}^{(2)}(t) f^{\prime}\left(m x+\frac{(1-t)}{n+1} \eta\left(a_{2}, m x\right)\right)}{\eta\left(a_{2}, m x\right)}\right|_{0} ^{1} \\
& -\frac{(n+1)}{\eta\left(a_{2}, m x\right)} \\
& \left.\times \int_{0}^{1} \Delta_{m, n}^{(2)}(t) f^{\prime}\left(m x+\frac{(1-t)}{n+1} \eta\left(a_{2}, m x\right)\right) d t\right\} \\
& =\frac{\eta^{2}\left(x, m a_{1}\right)}{2(n+1)^{2} \Lambda_{m, n}^{(1)}(1)} \\
& \times\left\{\frac{(n+1) \Lambda_{m, n}^{(1)}(1) f^{\prime}\left(m a_{1}+\eta\left(x, m a_{1}\right)\right)}{\eta\left(x, m a_{1}\right)}\right. \\
& -\frac{(n+1)}{\eta\left(x, m a_{1}\right)} \\
& \times\left[\frac{(n+1) \Delta_{m, n}^{(1)}(1) f\left(m a_{1}+\eta\left(x, m a_{1}\right)\right)}{\eta\left(x, m a_{1}\right)}\right. \\
& -\frac{(n+1)}{\eta\left(x, m a_{1}\right)} \\
& \left.\times_{\left(m a_{1}+\eta\left(x, m a_{1}\right)\right)^{-}} I_{\varphi} f\left(m a_{1}+\frac{n}{n+1} \eta\left(x, m a_{1}\right)\right)\right]
\end{aligned}
$$$$
+\frac{(n+1) \Lambda_{m, n}^{(1)}(1) f^{\prime}\left(m a_{1}\right)}{\eta\left(x, m a_{1}\right)}
$$$$
-\frac{(n+1)}{\eta\left(x, m a_{1}\right)} \times\left[\frac{(n+1) \Delta_{m, n}^{(1)}(1) f\left(m a_{1}\right)}{\eta\left(x, m a_{1}\right)}\right.
$$$$
\left.-\frac{(n+1)}{\eta\left(x, m a_{1}\right)} \times{ }_{\left(m a_{1}\right)}+I_{\varphi} f\left(m a_{1}+\frac{\eta\left(x, m a_{1}\right)}{n+1}\right)\right\}
$$$$
+\frac{\eta^{2}\left(a_{2}, m x\right)}{2(n+1)^{2} \Lambda_{m, n}^{(2)}(1)}
$$$$
\times\left\{\frac{(n+1) \Lambda_{m, n}^{(2)}(1) f^{\prime}\left(m x+\eta\left(a_{2}, m x\right)\right)}{\eta\left(a_{2}, m x\right)}\right.
$$$$
-\frac{(n+1)}{\eta\left(a_{2}, m x\right)}
$$$$
\times\left[\frac{(n+1) \Delta_{m, n}^{(2)}(1) f\left(m x+\eta\left(a_{2}, m x\right)\right)}{\eta\left(a_{2}, m x\right)}\right.
$$$$
-\frac{(n+1)}{\eta\left(a_{2}, m x\right)}
$$$$
\left.\times_{\left(m x+\eta\left(a_{2}, m x\right)\right)}-I_{\varphi} f\left(m x+\frac{n}{n+1} \eta\left(a_{2}, m x\right)\right)\right]
$$$$
+\frac{(n+1) \Lambda_{m, n}^{(2)}(1) f^{\prime}(m x)}{\eta\left(a_{2}, m x\right)}-\frac{(n+1)}{\eta\left(a_{2}, m x\right)}
$$ 


$$
\begin{gathered}
\times\left[\frac{(n+1) \Delta_{m, n}^{(2)}(1) f(m x)}{\eta\left(a_{2}, m x\right)}\right. \\
\left.-\frac{(n+1)}{\eta\left(a_{2}, m x\right)} \times{ }_{(m x)^{+}} I_{\varphi} f\left(m x+\frac{\eta\left(a_{2}, m x\right)}{n+1}\right)\right\} \\
=\frac{\eta\left(x, m a_{1}\right) \Lambda_{m, n}^{(1)}(1)}{(n+1) \Delta_{m, n}^{(1)}(1)} \\
\times \frac{f^{\prime}\left(m a_{1}\right)+f^{\prime}\left(m a_{1}+\eta\left(x, m a_{1}\right)\right)}{2} \\
-\frac{f\left(m a_{1}\right)+f\left(m a_{1}+\eta\left(x, m a_{1}\right)\right)}{2}-\frac{1}{2 \Delta_{m, n}^{(1)}(1)} \\
+\left[\begin{array}{c}
\left(m a_{1}\right)^{+} I_{\varphi} f\left(m a_{1}+\frac{\eta\left(x, m a_{1}\right)}{n+1}\right) \\
\left.+a_{1}+\eta\left(x, m a_{1}\right)\right)^{-} I_{\varphi} f\left(m a_{1}+\frac{n}{n+1} \eta\left(x, m a_{1}\right)\right)
\end{array}\right] \\
+\frac{\eta\left(a_{2}, m x\right) \Lambda_{m, n}^{(2)}(1)}{(n+1) \Delta_{m, n}^{(2)}(1)} \\
\times \frac{f^{\prime}(m x)+f^{\prime}\left(m x+\eta\left(a_{2}, m x\right)\right)}{2} \\
\times \frac{f(m x)+f\left(m x+\eta\left(a_{2}, m x\right)\right)}{2}-\frac{1}{2 \Delta_{m, n}^{(2)}(1)} \\
(m x)^{+} I_{\varphi} f\left(m x+\frac{\eta\left(a_{2}, m x\right)}{n+1}\right) \\
\left.+\frac{n}{n+m x))^{-} I_{\varphi} f\left(m x+\frac{n}{n+1} \eta\left(a_{2}, m x\right)\right)}\right] .
\end{gathered}
$$

The proof of Lemma 1 is completed.

Remark 1. Taking $m=1, n=0, x=$ $\frac{a_{1}+a_{2}}{2}, \eta\left(x, m a_{1}\right)=x-m a_{1}, \eta\left(a_{2}, m x\right)=a_{2}-m x$ and $\varphi(t)=t$ in Lemma 1, we get

$$
\begin{array}{r}
I_{f, \Lambda_{1,0}^{(1)}, \Lambda_{1,0}^{(2)}, \Delta_{1,0}^{(1)}, \Delta_{1,0}^{(2)}}\left(\frac{a_{1}+a_{2}}{2}, a_{1}, a_{2}\right) \\
=\left(\frac{a_{2}-a_{1}}{2}\right) \\
\times\left[\frac{f^{\prime}\left(a_{1}\right)+2 f^{\prime}\left(\frac{a_{1}+a_{2}}{2}\right)+f^{\prime}\left(a_{2}\right)}{2}\right] \\
-\left[\frac{f\left(a_{1}\right)+2 f\left(\frac{a_{1}+a_{2}}{2}\right)+f\left(a_{2}\right)}{2}\right] \\
-\frac{2}{\left(a_{2}-a_{1}\right)} \int_{a_{1}}^{a_{2}} f(t) d t .
\end{array}
$$

Theorem 2. Let $f: P \longrightarrow \mathbb{R}$ be a twice differentiable mapping on $\left(m a_{1}, a_{2}\right)$. If $\left|f^{\prime \prime}\right|^{q}$ is preinvex on $P$ for $q>1$ and $p^{-1}+q^{-1}=1$, then the following inequality for generalized fractional integrals hold:

$$
\left|I_{f, \Lambda_{m, n}^{(1)}, \Lambda_{m, n}^{(2)}, \Delta_{m, n}^{(1)}, \Delta_{m, n}^{(2)}}\left(x, a_{1}, a_{2}\right)\right|
$$

$$
\begin{gathered}
\leq \frac{\eta^{2}\left(x, m a_{1}\right)}{2(n+1)^{2} \sqrt[q]{2(n+1)} \Delta_{m, n}^{(1)}(1)} \\
\times \sqrt[p]{B_{\Lambda_{m, n}^{(1)}}(p)} \\
\times\left\{\sqrt[q]{\left|f^{\prime \prime}\left(m a_{1}\right)\right|^{q}+(2 n+1)\left|f^{\prime \prime}(x)\right|^{q}}\right. \\
\left.+\sqrt[q]{(2 n+1)\left|f^{\prime \prime}\left(m a_{1}\right)\right|^{q}+\left|f^{\prime \prime}(x)\right|^{q}}\right\} \\
+\frac{\eta^{2}\left(a_{2}, m x\right)}{2(n+1)^{2} \sqrt[q]{2(n+1)} \Delta_{m, n}^{(2)}(1)} \sqrt[p]{B_{\Lambda_{m, n}^{(2)}}(p)} \\
\times\left\{\sqrt[q]{\left|f^{\prime \prime}(m x)\right|^{q}+(2 n+1)\left|f^{\prime \prime}\left(a_{2}\right)\right|^{q}}\right. \\
\left.+\sqrt[q]{(2 n+1)\left|f^{\prime \prime}(m x)\right|^{q}+\left|f^{\prime \prime}\left(a_{2}\right)\right|^{q}}\right\}
\end{gathered}
$$

where

$$
B_{\Lambda_{m, n}^{(i)}}(p)=\int_{0}^{1}\left[\Lambda_{m, n}^{(i)}(t)\right]^{p} d t, \quad \forall i=1,2 .
$$

Proof. From Lemma 1, preinvexity of $\left|f^{\prime \prime}\right|^{q}$, Hölder's inequality and properties of the modulus, we have

$$
\begin{gathered}
|| I_{f, \Lambda_{m, n}^{(1)}, \Lambda_{m, n}^{(2)}, \Delta_{m, n}^{(1)}, \Delta_{m, n}^{(2)}\left(x, a_{1}, a_{2}\right) \mid} \leq \frac{\eta^{2}\left(x, m a_{1}\right)}{2(n+1)^{2} \Delta_{m, n}^{(1)}(1)} \\
\times\left\{\int _ { 0 } ^ { 1 } \Lambda _ { m , n } ^ { ( 1 ) } ( t ) \left[\left|f^{\prime \prime}\left(m a_{1}+\frac{(n+t)}{n+1} \eta\left(x, m a_{1}\right)\right)\right|\right.\right. \\
\left.\left.+\left|f^{\prime \prime}\left(m a_{1}+\frac{(1-t)}{n+1} \eta\left(x, m a_{1}\right)\right)\right|\right] d t\right\} \\
\times\left\{\int _ { 0 } ^ { 1 } \Lambda _ { m , n } ^ { ( 2 ) } ( t ) \left[\left|f^{\prime \prime}\left(m x+\frac{(1-t)}{n+1} \eta\left(a_{2}, m x\right)\right)\right|\right.\right. \\
\left.\left.+\left|f^{\prime \prime}\left(m x+\frac{(n+t)}{n+1} \eta\left(a_{2}, m x\right)\right)\right|\right] d t\right\} \\
\leq \frac{\eta^{2}\left(x, m a_{1}\right)}{2(n+1)^{2} \Delta_{m, n}^{(1)}(1)}\left(\int_{0}^{1}\left[\Lambda_{m, n}^{(2)}(t)\right]^{p} d t\right)^{\frac{1}{p}} \\
\times\left\{\left(\int_{0}^{1}\left|f^{\prime \prime}\left(m a_{1}+\frac{(n+t)}{n+1} \eta\left(x, m a_{1}\right)\right)\right|^{q} d t\right)^{\frac{1}{q}}\right. \\
+\left(\int_{0}^{1}\left|f^{\prime \prime}\left(m x+\frac{(1-t)}{n+1} \eta\left(a_{2}, m x\right)\right)\right|^{q} d t\right)^{\frac{1}{q}} \\
+\frac{\eta^{\prime}\left(a_{1}+\frac{(1-t)}{n+1} \eta\left(x, m a_{1}\right)\right)}{2(n+1)^{2} \Delta_{m, n}^{(2)}(1)}\left(\int_{0}^{1}\left[\Lambda_{m, n}^{(2)}(t)\right]^{p} d t\right)^{\frac{1}{p}}
\end{gathered}
$$




$$
\begin{aligned}
& \left.+\left(\int_{0}^{1}\left|f^{\prime \prime}\left(m x+\frac{(n+t)}{n+1} \eta\left(a_{2}, m x\right)\right)\right|^{q} d t\right)^{\frac{1}{q}}\right\} \\
& \leq \frac{\eta^{2}\left(x, m a_{1}\right)}{2(n+1)^{2} \Delta_{m, n}^{(1)}(1)} \sqrt[p]{B_{\Lambda_{m, n}^{(1)}}(p)} \\
& \times\left\{\left[\int _ { 0 } ^ { 1 } \left[\left(1-\frac{n+t}{n+1}\right)\left|f^{\prime \prime}\left(m a_{1}\right)\right|^{q}\right.\right.\right. \\
& \left.\left.+\frac{(n+t)}{n+1}\left|f^{\prime \prime}(x)\right|^{q}\right] d t\right]^{\frac{1}{q}} \\
& +\left[\int _ { 0 } ^ { 1 } \left[\left(1-\frac{1-t}{n+1}\right)\left|f^{\prime \prime}\left(m a_{1}\right)\right|^{q}\right.\right. \\
& \left.\left.\left.+\frac{(1-t)}{n+1}\left|f^{\prime \prime}(x)\right|^{q}\right] d t\right]^{\frac{1}{q}}\right\} \\
& +\frac{\eta^{2}\left(a_{2}, m x\right)}{2(n+1)^{2} \Delta_{m, n}^{(2)}(1)} \sqrt[p]{B_{\Lambda_{m, n}^{(2)}}(p)} \\
& \times\left\{\left[\int _ { 0 } ^ { 1 } \left[\left(1-\frac{1-t}{n+1}\right)\left|f^{\prime \prime}(m x)\right|^{q}\right.\right.\right. \\
& \left.\left.+\frac{(1-t)}{n+1}\left|f^{\prime \prime}\left(a_{2}\right)\right|^{q}\right] d t\right]^{\frac{1}{q}} \\
& +\left[\int _ { 0 } ^ { 1 } \left[\left(1-\frac{n+t}{n+1}\right)\left|f^{\prime \prime}(m x)\right|^{q}\right.\right. \\
& \left.\left.\left.+\frac{(n+t)}{n+1}\left|f^{\prime \prime}\left(a_{2}\right)\right|^{q}\right] d t\right]^{\frac{1}{q}}\right\} \\
& =\frac{\eta^{2}\left(x, m a_{1}\right)}{2(n+1)^{2} \sqrt[q]{2(n+1)} \Delta_{m, n}^{(1)}(1)} \sqrt[p]{B_{\Lambda_{m, n}^{(1)}}(p)} \\
& \times\left\{\sqrt[q]{\left|f^{\prime \prime}\left(m a_{1}\right)\right|^{q}+(2 n+1)\left|f^{\prime \prime}(x)\right|^{q}}\right. \\
& \left.+\sqrt[q]{(2 n+1)\left|f^{\prime \prime}\left(m a_{1}\right)\right|^{q}+\left|f^{\prime \prime}(x)\right|^{q}}\right\} \\
& +\frac{\eta^{2}\left(a_{2}, m x\right)}{2(n+1)^{2} \sqrt[q]{2(n+1)} \Delta_{m, n}^{(2)}(1)} \sqrt[p]{B_{\Lambda_{m, n}^{(2)}}(p)} \\
& \times\left\{\sqrt[q]{\left|f^{\prime \prime}(m x)\right|^{q}+(2 n+1)\left|f^{\prime \prime}\left(a_{2}\right)\right|^{q}}\right. \\
& \left.+\sqrt[q]{(2 n+1)\left|f^{\prime \prime}(m x)\right|^{q}+\mid f^{\prime \prime}\left(a_{2}\right)^{q}}\right\} \text {. }
\end{aligned}
$$

The proof of Theorem 2 is completed.

We point out some special cases of Theorem 2,

Corollary 1. Taking $p=q=2$ in Theorem [, we have

$$
\begin{gathered}
\left|I_{f, \Lambda_{m, n}^{(1)}, \Lambda_{m, n}^{(2)}, \Delta_{m, n}^{(1)}, \Delta_{m, n}^{(2)}}\left(x, a_{1}, a_{2}\right)\right| \\
\leq \frac{\eta^{2}\left(x, m a_{1}\right)}{2(n+1)^{2} \sqrt{2(n+1)} \Delta_{m, n}^{(1)}(1)} \\
\times \sqrt{B_{\Lambda_{m, n}^{(1)}}^{(1)}(2)}
\end{gathered}
$$

$$
\begin{aligned}
& \quad \times\left\{\sqrt{\left|f^{\prime \prime}\left(m a_{1}\right)\right|^{2}+(2 n+1)\left|f^{\prime \prime}(x)\right|^{2}}\right. \\
& \left.+\sqrt{(2 n+1)\left|f^{\prime \prime}\left(m a_{1}\right)\right|^{2}+\left|f^{\prime \prime}(x)\right|^{2}}\right\} \\
& +\frac{\eta^{2}\left(a_{2}, m x\right)}{2(n+1)^{2} \sqrt{2(n+1)} \Delta_{m, n}^{(2)}(1)} \sqrt{B_{\Lambda_{m, n}^{(2)}}(2)} \\
& \quad \times\left\{\sqrt{\left|f^{\prime \prime}(m x)\right|^{2}+(2 n+1)\left|f^{\prime \prime}\left(a_{2}\right)\right|^{2}}\right. \\
& \left.+\sqrt{(2 n+1)\left|f^{\prime \prime}(m x)\right|^{2}+\left|f^{\prime \prime}\left(a_{2}\right)\right|^{2}}\right\} .
\end{aligned}
$$

Corollary 2. Taking $\varphi(t)=t$ in Theorem 2, we get

$$
\begin{gathered}
\mid I_{f, \Lambda_{m, n}^{(1)}, \Lambda_{m, n}^{(2)}, \Delta_{m, n}^{(1)}, \Delta_{m, n}^{(2)}\left(x, a_{1}, a_{2}\right) \mid} \\
\leq \frac{\eta^{2}\left(x, m a_{1}\right)}{4(n+1) \sqrt[q]{2(n+1)} \sqrt[p]{2 p+1}} \\
\times\left\{\sqrt[q]{\left|f^{\prime \prime}\left(m a_{1}\right)\right|^{q}+(2 n+1)\left|f^{\prime \prime}(x)\right|^{q}}\right. \\
\left.+\sqrt[q]{(2 n+1)\left|f^{\prime \prime}\left(m a_{1}\right)\right|^{q}+\left|f^{\prime \prime}(x)\right|^{q}}\right\} \\
+\frac{\eta^{2}\left(a_{2}, m x\right)}{4(n+1)^{2} \sqrt[q]{2(n+1)} \sqrt[p]{2 p+1}} \\
\times\left\{\sqrt[q]{\left|f^{\prime \prime}(m x)\right|^{q}+(2 n+1)\left|f^{\prime \prime}\left(a_{2}\right)\right|^{q}}\right. \\
\left.+\sqrt[q]{(2 n+1)\left|f^{\prime \prime}(m x)\right|^{q}+\left|f^{\prime \prime}\left(a_{2}\right)\right|^{q}}\right\} .
\end{gathered}
$$

Corollary 3. Taking $x=\frac{a_{1}+a_{2}}{2}, m=1, n=$ $0, \eta\left(x, m a_{1}\right)=x-m a_{1}$ and $\eta\left(a_{2}, m x\right)=a_{2}-m x$ in Corollary 2 , we obtain

$$
\begin{gathered}
\left|I_{f, \Lambda_{1,0}^{(1)}, \Lambda_{1,0}^{(2)}, \Delta_{1,0}^{(1)}, \Delta_{1,0}^{(2)}}\left(\frac{a_{1}+a_{2}}{2}, a_{1}, a_{2}\right)\right| \\
\leq \frac{\left(a_{2}-a_{1}\right)^{2}}{8 \sqrt[q]{2} \sqrt[p]{2 p+1}} \\
\times\left\{\sqrt[q]{\left|f^{\prime \prime}\left(a_{1}\right)\right|^{q}+\left|f^{\prime \prime}\left(\frac{a_{1}+a_{2}}{2}\right)\right|^{q}}\right. \\
+\sqrt[q]{\left.\left|f^{\prime \prime}\left(\frac{a_{1}+a_{2}}{2}\right)\right|^{q}+\left|f^{\prime \prime}\left(a_{2}\right)\right|^{q}\right\} .}
\end{gathered}
$$

Corollary 4. Taking $\varphi(t)=\frac{t^{\alpha}}{\Gamma(\alpha)}$ in Theorem 2 , we have

$$
\begin{aligned}
& \left|I_{f, \Lambda_{m, n}^{(1)}, \Lambda_{m, n}^{(2)}, \Delta_{m, n}^{(1)}, \Delta_{m, n}^{(2)}}\left(x, a_{1}, a_{2}\right)\right| \\
\leq & \frac{\eta^{2}\left(x, m a_{1}\right)}{4(n+1)^{2} \sqrt[q]{2(n+1)} \sqrt[p]{2 p \alpha+1}} \\
\times & \left\{\sqrt[q]{\left|f^{\prime \prime}\left(m a_{1}\right)\right|^{q}+(2 n+1)\left|f^{\prime \prime}(x)\right|^{q}}\right. \\
+ & \left.\sqrt[q]{(2 n+1)\left|f^{\prime \prime}\left(m a_{1}\right)\right|^{q}+\left|f^{\prime \prime}(x)\right|^{q}}\right\} \\
& +\frac{\eta^{2}\left(a_{2}, m x\right)}{4(n+1)^{2} \sqrt[q]{2(n+1)} \sqrt[p]{2 p \alpha+1}} \\
& \times\left\{\sqrt[q]{\left|f^{\prime \prime}(m x)\right|^{q}+(2 n+1)\left|f^{\prime \prime}\left(a_{2}\right)\right|^{q}}\right.
\end{aligned}
$$




$$
\left.+\sqrt[q]{(2 n+1)\left|f^{\prime \prime}(m x)\right|^{q}+\left|f^{\prime \prime}\left(a_{2}\right)\right|^{q}}\right\} .
$$

Corollary 5. Taking $\varphi(t)=\frac{t^{\frac{\alpha}{k}}}{k \Gamma_{k}(\alpha)}$ in Theorem [2, we get

$$
\begin{aligned}
& \left|I_{f, \Lambda_{m, n}^{(1)}, \Lambda_{m, n}^{(2)}, \Delta_{m, n}^{(1)}, \Delta_{m, n}^{(2)}}\left(x, a_{1}, a_{2}\right)\right| \\
\leq & \frac{\eta^{2}\left(x, m a_{1}\right)}{4(n+1)^{2} \sqrt[q]{2(n+1)} \sqrt[p]{\frac{2 p \alpha}{k}+1}} \\
\times & \left\{\sqrt[q]{\left|f^{\prime \prime}\left(m a_{1}\right)\right|^{q}+(2 n+1)\left|f^{\prime \prime}(x)\right|^{q}}\right. \\
+ & \left.\sqrt[q]{(2 n+1)\left|f^{\prime \prime}\left(m a_{1}\right)\right|^{q}+\left|f^{\prime \prime}(x)\right|^{q}}\right\} \\
& +\frac{\eta^{2}\left(a_{2}, m x\right)}{4(n+1)^{2} \sqrt[q]{2(n+1)} \sqrt[p]{\frac{2 p \alpha}{k}+1}} \\
& \times\left\{\sqrt[q]{\left|f^{\prime \prime}(m x)\right|^{q}+(2 n+1)\left|f^{\prime \prime}\left(a_{2}\right)\right|^{q}}\right. \\
+ & \left.\sqrt[q]{(2 n+1)\left|f^{\prime \prime}(m x)\right|^{q}+\left|f^{\prime \prime}\left(a_{2}\right)\right|^{q}}\right\} .
\end{aligned}
$$

Corollary 6. Taking $\varphi(t)=t\left(a_{2}-t\right)^{\alpha-1}$ and $f(x)$ is symmetric to $x=\frac{m a_{1}+a_{2}}{2}$, in Theorem 2 , we obtain

$$
\begin{aligned}
& \left|I_{f, \Lambda_{m, n}^{(1)}, \Lambda_{m, n}^{(2)}, \Delta_{m, n}^{(1)}, \Delta_{m, n}^{(2)}}\left(\frac{m a_{1}+a_{2}}{2}, a_{1}, a_{2}\right)\right|
\end{aligned}
$$

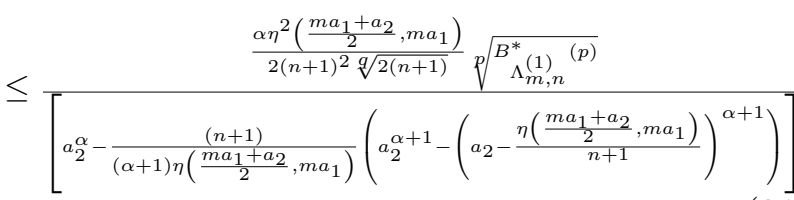

$$
\begin{aligned}
& \times\left\{\sqrt[q]{\left|f^{\prime \prime}\left(m a_{1}\right)\right|^{q}+(2 n+1)\left|f^{\prime \prime}\left(\frac{m a_{1}+a_{2}}{2}\right)\right|^{q}}\right. \\
& \left.+\sqrt[q]{(2 n+1)\left|f^{\prime \prime}\left(m a_{1}\right)\right|^{q}+\left|f^{\prime \prime}\left(\frac{m a_{1}+a_{2}}{2}\right)\right|^{q}}\right\} \\
& \frac{\alpha \eta^{2}\left(a_{2}, m \frac{\left(m a_{1}+a_{2}\right)}{2}\right)}{2(n+1)^{2} \sqrt[q]{2(n+1)}} p \sqrt{B_{\Lambda_{m, n}^{*}(2)}^{(p)}} \\
& +\left[a_{2}^{\alpha}-\frac{(n+1)}{(\alpha+1) \eta\left(a_{2}, m \frac{\left(m a_{1}+a_{2}\right)}{2}\right)}\left(a_{2}^{\alpha+1}-\left(a_{2}-\frac{\eta\left(a_{2}, m \frac{\left(m a_{1}+a_{2}\right)}{n+1}\right)}{n+1}\right)\right]\right. \\
& \times\left\{\sqrt[q]{\left|f^{\prime \prime}\left(m \frac{\left(m a_{1}+a_{2}\right)}{2}\right)\right|^{q}+(2 n+1)\left|f^{\prime \prime}\left(a_{2}\right)\right|^{q}}\right. \\
& +\sqrt[q]{\left.(2 n+1)\left|f^{\prime \prime}\left(m \frac{\left(m a_{1}+a_{2}\right)}{2}\right)\right|^{q}+\left|f^{\prime \prime}\left(a_{2}\right)\right|^{q}\right\}} \text {, } \\
& \text { where } \\
& B_{\Lambda_{m, n}^{(1)}}^{*}(p)=\frac{1}{\alpha} \\
& \times \int_{0}^{1}\left[a_{2}^{\alpha} t-\frac{(n+1)}{(\alpha+1) \eta\left(\frac{m a_{1}+a_{2}}{2}, m a_{1}\right)}\right.
\end{aligned}
$$

$$
\left.\times\left(a_{2}^{\alpha+1}-\left(a_{2}-\frac{\eta\left(\frac{m a_{1}+a_{2}}{2}, m a_{1}\right) t}{n+1}\right)^{\alpha+1}\right)\right]^{p} d t
$$

and

$$
\begin{gathered}
B_{\Lambda_{m, n}^{(2)}}^{*}(p)=\frac{1}{\alpha} \\
\times \int_{0}^{1}\left[a_{2}^{\alpha} t-\frac{(n+1)}{(\alpha+1) \eta\left(a_{2}, m \frac{\left(m a_{1}+a_{2}\right)}{2}\right)}\right. \\
\left.\times\left(a_{2}^{\alpha+1}-\left(a_{2}-\frac{\eta\left(a_{2}, m \frac{\left(m a_{1}+a_{2}\right)}{2}\right) t}{n+1}\right)^{\alpha+1}\right)\right]^{p} d t .
\end{gathered}
$$

Corollary 7. Taking $\varphi(t)=\frac{t}{\alpha} \exp \left[\left(-\frac{1-\alpha}{\alpha}\right) t\right]$ for $\alpha \in(0,1)$, in Theorem Q, we have

$$
\begin{gathered}
\left|I_{f, \Lambda_{m, n}^{(1)}, \Lambda_{m, n}^{(2)}, \Delta_{m, n}^{(1)}, \Delta_{m, n}^{(2)}}\left(x, a_{1}, a_{2}\right)\right| \\
\leq \frac{(\alpha-1) \eta^{2}\left(x, m a_{1}\right)}{2(n+1)^{2} \sqrt[q]{2(n+1)}}
\end{gathered}
$$

$$
\times \frac{1}{\left\{\exp \left[\left(-\frac{1-\alpha}{\alpha}\right) \frac{\eta\left(x, m a_{1}\right)}{n+1}\right]-1\right\}}
$$$$
\times p \sqrt{B_{\Lambda_{m, n}^{(1)}}^{\diamond}(p)}
$$$$
\times\left\{\sqrt[q]{\left|f^{\prime \prime}\left(m a_{1}\right)\right|^{q}+(2 n+1)\left|f^{\prime \prime}(x)\right|^{q}}\right.
$$$$
\left.+\sqrt[q]{(2 n+1)\left|f^{\prime \prime}\left(m a_{1}\right)\right|^{q}+\left|f^{\prime \prime}(x)\right|^{q}}\right\}
$$

$$
\begin{gathered}
+\frac{(\alpha-1) \eta^{2}\left(a_{2}, m x\right)}{2(n+1)^{2} \sqrt[q]{2(n+1)}\left\{\exp \left[\left(-\frac{1-\alpha}{\alpha}\right) \frac{\eta\left(a_{2}, m x\right)}{n+1}\right]-1\right\}} \\
\times \sqrt[p]{B_{\Lambda_{m, n}^{(2)}}^{\diamond}(p)} \\
+\sqrt[q]{\left|f^{\prime \prime}(m x)\right|^{q}+(2 n+1)\left|f^{\prime \prime}\left(a_{2}\right)\right|^{q}} \\
\left.+\sqrt[q]{(2 n+1)\left|f^{\prime \prime}(m x)\right|^{q}+\left|f^{\prime \prime}\left(a_{2}\right)\right|^{q}}\right\}
\end{gathered}
$$

where

$$
\begin{gathered}
B_{\Lambda_{m, n}^{(1)}}(p)=\frac{1}{(\alpha-1)^{p}} \\
\times \int_{0}^{1}\left[\frac{(n+1) \alpha}{(\alpha-1) \eta\left(x, m a_{1}\right)}\right.
\end{gathered}
$$

$\left.\times\left\{\exp \left[\left(-\frac{1-\alpha}{\alpha}\right) \frac{\eta\left(x, m a_{1}\right)}{(n+1)} t\right]-(t+1)\right\}\right]^{p} d t$ and

$$
\begin{gathered}
B_{\Lambda_{m, n}^{(2)}}(p)=\frac{1}{(\alpha-1)^{p}} \\
\times \int_{0}^{1}\left[\frac{(n+1) \alpha}{(\alpha-1) \eta\left(a_{2}, m x\right)}\right.
\end{gathered}
$$


$\left.\times\left\{\exp \left[\left(-\frac{1-\alpha}{\alpha}\right) \frac{\eta\left(a_{2}, m x\right)}{(n+1)} t\right]-(t+1)\right\}\right]^{p} d t$.

Theorem 3. Let $f: P \longrightarrow \mathbb{R}$ be a twice differentiable mapping on $\left(m a_{1}, a_{2}\right)$. If $\left|f^{\prime \prime}\right|^{q}$ is preinvex on $P$ for $q \geq 1$, then the following inequality for generalized fractional integrals hold:

$$
\begin{gathered}
\left|I_{f, \Lambda_{m, n}^{(1)}, \Lambda_{m, n}^{(2)}, \Delta_{m, n}^{(1)}, \Delta_{m, n}^{(2)}}\left(x, a_{1}, a_{2}\right)\right| \\
\leq\left(\frac{1}{n+1}\right)^{\frac{2 q+1}{q}} \frac{\eta^{2}\left(x, m a_{1}\right)}{2 \Delta_{m, n}^{(1)}(1)} \\
\times\left(B_{\Lambda_{m, n}^{(1)}}(1)\right)^{1-\frac{1}{q}} \\
\times\left\{\sqrt[q]{C_{\Lambda_{m, n}^{(1)}}^{(1)}\left|f^{\prime \prime}\left(m a_{1}\right)\right|^{q}+D_{\Lambda_{m, n}^{(1)}}(n)\left|f^{\prime \prime}(x)\right|^{q}}\right. \\
\left.+\sqrt[q]{D_{\Lambda_{m, n}^{(1)}}(n)\left|f^{\prime \prime}\left(m a_{1}\right)\right|^{q}+C_{\Lambda_{m, n}^{(1)}}\left|f^{\prime \prime}(x)\right|^{q}}\right\} \\
+\left(\frac{1}{n+1}\right)^{\frac{2 q+1}{q}} \frac{\eta^{2}\left(a_{2}, m x\right)}{2 \Delta_{m, n}^{(2)}(1)}\left(B_{\Lambda_{m, n}^{(2)}}(1)\right)^{1-\frac{1}{q}} \\
\times\left\{\sqrt[q]{C_{\Lambda_{m, n}^{(2)}}\left|f^{\prime \prime}(m x)\right|^{q}+D_{\Lambda_{m, n}^{(2)}}(n)\left|f^{\prime \prime}\left(a_{2}\right)\right|^{q}}\right. \\
\left.+\sqrt[q]{D_{\Lambda_{m, n}^{(2)}}(n)\left|f^{\prime \prime}(m x)\right|^{q}+C_{\Lambda_{m, n}^{(2)}}\left|f^{\prime \prime}\left(a_{2}\right)\right|^{q}}\right\},
\end{gathered}
$$

where

$$
\begin{gathered}
C_{\Lambda_{m, n}^{(i)}}=\int_{0}^{1}(1-t) \Lambda_{m, n}^{(i)}(t) d t, \quad \forall i=1,2 \\
D_{\Lambda_{m, n}^{(i)}}(n)=\int_{0}^{1}(n+t) \Lambda_{m, n}^{(i)}(t) d t, \forall i=1,2
\end{gathered}
$$

and $B_{\Lambda_{m, n}^{(i)}}(1), \forall i=1,2$, are defined as in Theorem 2 , where $p=1$.

Proof. From Lemma 1, preinvexity of $\left|f^{\prime \prime}\right|^{q}$, power mean inequality and properties of the modulus, we have

$$
\begin{gathered}
\left|I_{f, \Lambda_{m, n}^{(1)}, \Lambda_{m, n}^{(2)}, \Delta_{m, n}^{(1)}, \Delta_{m, n}^{(2)}}\left(x, a_{1}, a_{2}\right)\right| \\
\leq \frac{\eta^{2}\left(x, m a_{1}\right)}{2(n+1)^{2} \Delta_{m, n}^{(1)}(1)} \\
\times\left\{\int _ { 0 } ^ { 1 } \Lambda _ { m , n } ^ { ( 1 ) } ( t ) \left[\left|f^{\prime \prime}\left(m a_{1}+\frac{(n+t)}{n+1} \eta\left(x, m a_{1}\right)\right)\right|\right.\right. \\
\left.\left.+\left|f^{\prime \prime}\left(m a_{1}+\frac{(1-t)}{n+1} \eta\left(x, m a_{1}\right)\right)\right|\right] d t\right\} \\
\times \frac{\eta^{2}\left(a_{2}, m x\right)}{2(n+1)^{2} \Delta_{m, n}^{(2)}(1)} \\
\times\left\{\int _ { 0 } ^ { 1 } \Lambda _ { m , n } ^ { ( 2 ) } ( t ) \left[\left|f^{\prime \prime}\left(m x+\frac{(1-t)}{n+1} \eta\left(a_{2}, m x\right)\right)\right|\right.\right.
\end{gathered}
$$

$$
\begin{aligned}
& \left.\left.+\left|f^{\prime \prime}\left(m x+\frac{(n+t)}{n+1} \eta\left(a_{2}, m x\right)\right)\right|\right] d t\right\} \\
& \leq \frac{\eta^{2}\left(x, m a_{1}\right)}{2(n+1)^{2} \Delta_{m, n}^{(1)}(1)}\left(\int_{0}^{1} \Lambda_{m, n}^{(1)}(t) d t\right)^{1-\frac{1}{q}} \\
& \times\left\{\left(\int_{0}^{1} \Lambda_{m, n}^{(1)}(t)\left|f^{\prime \prime}\left(m a_{1}+\frac{(n+t)}{n+1} \eta\left(x, m a_{1}\right)\right)\right|^{q} d t\right)^{\frac{1}{q}}\right. \\
& \left.+\left(\int_{0}^{1} \Lambda_{m, n}^{(1)}(t)\left|f^{\prime \prime}\left(m a_{1}+\frac{(1-t)}{n+1} \eta\left(x, m a_{1}\right)\right)\right|^{q} d t\right)^{\frac{1}{q}}\right\} \\
& +\frac{\eta^{2}\left(a_{2}, m x\right)}{2(n+1)^{2} \Delta_{m, n}^{(2)}(1)}\left(\int_{0}^{1} \Lambda_{m, n}^{(2)}(t) d t\right)^{1-\frac{1}{q}} \\
& \times\left\{\left(\int_{0}^{1} \Lambda_{m, n}^{(2)}(t)\left|f^{\prime \prime}\left(m x+\frac{(1-t)}{n+1} \eta\left(a_{2}, m x\right)\right)\right|^{q} d t\right)^{\frac{1}{q}}\right. \\
& \left.+\left(\int_{0}^{1} \Lambda_{m, n}^{(2)}(t)\left|f^{\prime \prime}\left(m x+\frac{(n+t)}{n+1} \eta\left(a_{2}, m x\right)\right)\right|^{q} d t\right)^{\frac{1}{q}}\right\} \\
& \leq \frac{\eta^{2}\left(x, m a_{1}\right)}{2(n+1)^{2} \Delta_{m, n}^{(1)}(1)}\left(B_{\Lambda_{m, n}^{(1)}}(1)\right)^{1-\frac{1}{q}} \\
& \times\left\{\left[\int_{0}^{1} \Lambda_{m, n}^{(1)}(t)\right.\right. \\
& \left.\times\left[\left(1-\frac{n+t}{n+1}\right)\left|f^{\prime \prime}\left(m a_{1}\right)\right|^{q}+\frac{(n+t)}{n+1}\left|f^{\prime \prime}(x)\right|^{q}\right] d t\right]^{\frac{1}{q}} \\
& +\left[\int_{0}^{1} \Lambda_{m, n}^{(1)}(t)\right. \\
& \left.\left.\times\left[\left(1-\frac{1-t}{n+1}\right)\left|f^{\prime \prime}\left(m a_{1}\right)\right|^{q}+\frac{(1-t)}{n+1}\left|f^{\prime \prime}(x)\right|^{q}\right] d t\right]^{\frac{1}{q}}\right\} \\
& +\frac{\eta^{2}\left(a_{2}, m x\right)}{2(n+1)^{2} \Delta_{m, n}^{(2)}(1)}\left(B_{\Lambda_{m, n}^{(2)}}(1)\right)^{1-\frac{1}{q}} \\
& \times\left\{\left[\int_{0}^{1} \Lambda_{m, n}^{(2)}(t)\right.\right. \\
& \left.\times\left[\left(1-\frac{1-t}{n+1}\right)\left|f^{\prime \prime}(m x)\right|^{q}+\frac{(1-t)}{n+1}\left|f^{\prime \prime}\left(a_{2}\right)\right|^{q}\right] d t\right]^{\frac{1}{q}} \\
& +\left[\int_{0}^{1} \Lambda_{m, n}^{(2)}(t)\right. \\
& \left.\left.\times\left[\left(1-\frac{n+t}{n+1}\right)\left|f^{\prime \prime}(m x)\right|^{q}+\frac{(n+t)}{n+1}\left|f^{\prime \prime}\left(a_{2}\right)\right|^{q}\right] d t\right]^{\frac{1}{q}}\right\} \\
& =\left(\frac{1}{n+1}\right)^{\frac{2 q+1}{q}} \frac{\eta^{2}\left(x, m a_{1}\right)}{2 \Delta_{m, n}^{(1)}(1)}\left(B_{\Lambda_{m, n}^{(1)}}(1)\right)^{1-\frac{1}{q}} \\
& \times\left\{\sqrt[q]{C_{\Lambda_{m, n}^{(1)}}\left|f^{\prime \prime}\left(m a_{1}\right)\right|^{q}+D_{\Lambda_{m, n}^{(1)}}(n)\left|f^{\prime \prime}(x)\right|^{q}}\right.
\end{aligned}
$$




$$
\begin{aligned}
& \left.+\sqrt[q]{D_{\Lambda_{m, n}^{(1)}}(n)\left|f^{\prime \prime}\left(m a_{1}\right)\right|^{q}+C_{\Lambda_{m, n}^{(1)}}\left|f^{\prime \prime}(x)\right|^{q}}\right\} \\
& +\left(\frac{1}{n+1}\right)^{\frac{2 q+1}{q}} \frac{\eta^{2}\left(a_{2}, m x\right)}{2 \Delta_{m, n}^{(2)}(1)}\left(B_{\Lambda_{m, n}^{(2)}}(1)\right)^{1-\frac{1}{q}} \\
& \times\left\{\sqrt[q]{C_{\Lambda_{m, n}^{(2)}}\left|f^{\prime \prime}(m x)\right|^{q}+D_{\Lambda_{m, n}^{(2)}}(n) \mid f^{\prime \prime}\left(a_{2}\right)^{q}}\right. \\
& \left.+\sqrt[q]{D_{\Lambda_{m, n}^{(2)}}(n)\left|f^{\prime \prime}(m x)\right|^{q}+C_{\Lambda_{m, n}^{(2)}} \mid f^{\prime \prime}\left(a_{2}\right)^{q}}\right\} .
\end{aligned}
$$

The proof of Theorem 3 is completed.

We point out some special cases of Theorem 3 ,

Corollary 8. Taking $q=1$ in Theorem 3, we have

$$
\begin{gathered}
\left|I_{f, \Lambda_{m, n}^{(1)}, \Lambda_{m, n}^{(2)}, \Delta_{m, n}^{(1)}, \Delta_{m, n}^{(2)}}\left(x, a_{1}, a_{2}\right)\right| \\
\leq \frac{1}{(n+1)^{3}}\left\{\frac{\eta^{2}\left(x, m a_{1}\right)}{2 \Delta_{m, n}^{(1)}(1)}\right. \\
\times\left(C_{\Lambda_{m, n}^{(1)}}+D_{\Lambda_{m, n}^{(1)}}(n)\right)\left[\left|f^{\prime \prime}\left(m a_{1}\right)\right|+\left|f^{\prime \prime}(x)\right|\right] \\
+\frac{\eta^{2}\left(a_{2}, m x\right)}{2 \Delta_{m, n}^{(2)}(1)} \\
\times\left(C_{\Lambda_{m, n}^{(2)}}+D_{\left.\Lambda_{m, n}^{(2)}(n)\right)}\left(\left|f^{\prime \prime}(m x)\right|+\left|f^{\prime \prime}\left(a_{2}\right)\right|\right]\right\} .
\end{gathered}
$$

Corollary 9. Taking $\varphi(t)=t$ in Theorem 3, we get

$$
\begin{gathered}
\mid I_{f, \Lambda_{m, n}^{(1)}, \Lambda_{m, n}^{(2)}, \Delta_{m, n}^{(1)}, \Delta_{m, n}^{(2)}\left(x, a_{1}, a_{2}\right) \mid} \\
\leq\left(\frac{1}{n+1}\right)^{\frac{2 q+1}{q}} \frac{\eta^{2}\left(x, m a_{1}\right)}{12 \sqrt[q]{4}} \\
\times\left\{\sqrt[q]{\left|f^{\prime \prime}\left(m a_{1}\right)\right|^{q}+(4 n+3)\left|f^{\prime \prime}(x)\right|^{q}}\right. \\
\left.+\sqrt[q]{(4 n+3)\left|f^{\prime \prime}\left(m a_{1}\right)\right|^{q}+\left|f^{\prime \prime}(x)\right|^{q}}\right\} \\
+\frac{\eta^{2}\left(a_{2}, m x\right)}{12 \sqrt[q]{4}} \\
\times\left\{\sqrt[q]{\left|f^{\prime \prime}(m x)\right|^{q}+(4 n+3)\left|f^{\prime \prime}\left(a_{2}\right)\right|^{q}}\right. \\
\left.+\sqrt[q]{(4 n+3)\left|f^{\prime \prime}(m x)\right|^{q}+\left|f^{\prime \prime}\left(a_{2}\right)\right|^{q}}\right\} .
\end{gathered}
$$

Corollary 10. Taking $x=\frac{a_{1}+a_{2}}{2}, m=1, n=$ $0, \eta\left(x, m a_{1}\right)=x-m a_{1}$ and $\eta\left(a_{2}, m x\right)=a_{2}-m x$ in Corollary 9, we obtain

$$
\begin{gathered}
\left|I_{f, \Lambda_{1,0}^{(1)}, \Lambda_{1,0}^{(2)}, \Delta_{1,0}^{(1)}, \Delta_{1,0}^{(2)}}\left(\frac{a_{1}+a_{2}}{2}, a_{1}, a_{2}\right)\right| \\
\leq\left(\frac{1}{n+1}\right)^{\frac{2 q+1}{q}} \frac{\left(a_{2}-a_{1}\right)^{2}}{48 \sqrt[q]{4}} \\
\quad \times\left\{\sqrt[q]{\left|f^{\prime \prime}\left(a_{1}\right)\right|^{q}+3\left|f^{\prime \prime}\left(\frac{a_{1}+a_{2}}{2}\right)\right|^{q}}\right.
\end{gathered}
$$

$$
\begin{aligned}
& +\sqrt[q]{3\left|f^{\prime \prime}\left(a_{1}\right)\right|^{q}+\left|f^{\prime \prime}\left(\frac{a_{1}+a_{2}}{2}\right)\right|^{q}} \\
& +\sqrt[q]{\left|f^{\prime \prime}\left(\frac{a_{1}+a_{2}}{2}\right)\right|^{q}+3\left|f^{\prime \prime}\left(a_{2}\right)\right|^{q}} \\
& +\sqrt[q]{\left.3\left|f^{\prime \prime}\left(\frac{a_{1}+a_{2}}{2}\right)\right|^{q}+\left|f^{\prime \prime}\left(a_{2}\right)\right|^{q}\right\} .}
\end{aligned}
$$

Corollary 11. Taking $\varphi(t)=\frac{t^{\alpha}}{\Gamma(\alpha)}$ in Theorem 3, we have

$$
\begin{gathered}
\left|I_{f, \Lambda_{m, n}^{(1)}, \Lambda_{m, n}^{(2)}, \Delta_{m, n}^{(1)}, \Delta_{m, n}^{(2)}}\left(x, a_{1}, a_{2}\right)\right| \\
\leq\left(\frac{1}{n+1}\right)^{\frac{2 q+1}{q}} \\
\times \frac{\Gamma(\alpha+1)}{2 \Gamma(\alpha+3)} \sqrt[q]{\frac{\Gamma(\alpha+3)}{\Gamma(\alpha+4)}} \eta^{2}\left(x, m a_{1}\right) \\
\times\left\{\sqrt[q]{\left|f^{\prime \prime}\left(m a_{1}\right)\right|^{q}+[n(\alpha+3)+(\alpha+2)]\left|f^{\prime \prime}(x)\right|^{q}}\right. \\
\left.+\sqrt[q]{[n(\alpha+3)+(\alpha+2)]\left|f^{\prime \prime}\left(m a_{1}\right)\right|^{q}+\left|f^{\prime \prime}(x)\right|^{q}}\right\} \\
+\left(\frac{1}{n+1}\right)^{\frac{2 q+1}{q}} \frac{\Gamma(\alpha+1)}{2 \Gamma(\alpha+3)} \sqrt[q]{\frac{\Gamma(\alpha+3)}{\Gamma(\alpha+4)}} \eta^{2}\left(a_{2}, m x\right) \\
\times\left\{\sqrt[q]{\left|f^{\prime \prime}(m x)\right|^{q}+[n(\alpha+3)+(\alpha+2)]\left|f^{\prime \prime}\left(a_{2}\right)\right|^{q}}\right. \\
\left.+\sqrt[q]{[n(\alpha+3)+(\alpha+2)]\left|f^{\prime \prime}(m x)\right|^{q}+\left|f^{\prime \prime}\left(a_{2}\right)\right|^{q}}\right\} .
\end{gathered}
$$

Corollary 12. Taking $\varphi(t)=\frac{t^{\frac{\alpha}{k}}}{k \Gamma_{k}(\alpha)}$ in Theorem 3. we get

$$
\begin{gathered}
\mid I_{f, \Lambda_{m, n}^{(1)}, \Lambda_{m, n}^{(2)}, \Delta_{m, n}^{(1)}, \Delta_{m, n}^{(2)}\left(x, a_{1}, a_{2}\right) \mid} \leq\left(\frac{1}{n+1}\right)^{\frac{2 q+1}{q}} \\
\times \frac{\Gamma_{k}(\alpha+k)}{2 \Gamma_{k}(\alpha+k+2)} \sqrt[q]{\frac{\Gamma_{k}(\alpha+k+2)}{\Gamma_{k}(\alpha+k+3)}} \eta^{2}\left(x, m a_{1}\right) \\
\times\left\{\sqrt[q]{\left|f^{\prime \prime}\left(m a_{1}\right)\right|^{q}+\left[n\left(\frac{\alpha}{k}+3\right)+\left(\frac{\alpha}{k}+2\right)\right]\left|f^{\prime \prime}(x)\right|^{q}}\right. \\
\left.+\sqrt[q]{\left[n\left(\frac{\alpha}{k}+3\right)+\left(\frac{\alpha}{k}+2\right)\right]\left|f^{\prime \prime}\left(m a_{1}\right)\right|^{q}+\left|f^{\prime \prime}(x)\right|^{q}}\right\} \\
\times \frac{\Gamma_{k}(\alpha+k)}{2 \Gamma_{k}(\alpha+k+2)} \sqrt[q]{\frac{\Gamma_{k}(\alpha+k+2)}{\Gamma_{k}(\alpha+k+3)}} \eta^{2}\left(a_{2}, m x\right)
\end{gathered}
$$


$\times\left\{\sqrt[q]{\left|f^{\prime \prime}(m x)\right|^{q}+\left[n\left(\frac{\alpha}{k}+3\right)+\left(\frac{\alpha}{k}+2\right)\right]\left|f^{\prime \prime}\left(a_{2}\right)\right|^{q}}\right.$
$+\sqrt[q]{\left.\left[n\left(\frac{\alpha}{k}+3\right)+\left(\frac{\alpha}{k}+2\right)\right]\left|f^{\prime \prime}(m x)\right|^{q}+\left|f^{\prime \prime}\left(a_{2}\right)\right|^{q}\right\}}$.

Remark 2. Applying our Theorems 2 and 3, for $n \in \mathbb{N}^{*}$ and appropriate choices of function $\varphi(t)=$ $t ; \varphi(t)=\frac{t^{\alpha}}{\Gamma(\alpha)} ; \frac{t^{\frac{\alpha}{k}}}{k \Gamma_{k}(\alpha)} ; \varphi(t)=t\left(a_{2}-t\right)^{\alpha-1}$, where $f(x)$ is symmetric to $x=\frac{m a_{1}+a_{2}}{2}$ and $m \in(0,1]$ is a fixed number; $\varphi(t)=\frac{t}{\alpha} \exp \left[\left(-\frac{1-\alpha}{\alpha}\right) t\right]$, for $\alpha \in(0,1)$; such that $\eta\left(x, m a_{1}\right)=x-m a_{1}$ and $\eta\left(a_{2}, m x\right)=a_{2}-m x, \forall x \in P$, we can deduce some new general fractional integral inequalities. We omit their proofs and the details are left to the interested readers.

\section{Applications to special means}

Consider the following special means for different real numbers $\alpha, \beta$ and $\alpha \beta \neq 0$, as follows:

(1) The arithmetic mean:

$$
A:=A(\alpha, \beta)=\frac{\alpha+\beta}{2},
$$

(2) The harmonic mean:

$$
H:=H(\alpha, \beta)=\frac{2}{\frac{1}{\alpha}+\frac{1}{\beta}},
$$

(3) The logarithmic mean:

$$
L:=L(\alpha, \beta)=\frac{\beta-\alpha}{\ln |\beta|-\ln |\alpha|},
$$

(4) The generalized $\log -$ mean:

$$
\begin{aligned}
& L_{r}:=L_{r}(\alpha, \beta)=\left[\frac{\beta^{r+1}-\alpha^{r+1}}{(r+1)(\beta-\alpha)}\right]^{\frac{1}{r}}, \\
& \text { where } r \in \mathbb{Z} \backslash\{-1,0\} .
\end{aligned}
$$

It is well known that $L_{r}$ is monotonic nondecreasing over $r \in \mathbb{Z}$ with $L_{-1}:=L$. In particular, we have the following inequality $H \leq L \leq A$. Now, using the theory results in section 2 , we give some applications to special means for different real numbers.

Proposition 1. Let $a_{1}, a_{2} \in \mathbb{R} \backslash\{0\}$, where $a_{1}<a_{2}$ and $x \in\left[a_{1}, a_{2}\right]$. Then for $r \in\{2,3, \ldots\}$, where $q>1$ and $p^{-1}+q^{-1}=1$, the following inequality hold:

$$
\begin{aligned}
& \mid r\left(\frac{a_{2}-a_{1}}{2}\right)\left[A\left(a_{1}^{r-1}, a_{2}^{r-1}\right)+A^{r-1}\left(a_{1}, a_{2}\right)\right] \\
& \quad-\left[A\left(a_{1}^{r}, a_{2}^{r}\right)+A^{r}\left(a_{1}, a_{2}\right)\right]-2 L_{r}^{r}\left(a_{1}, a_{2}\right) \mid
\end{aligned}
$$

$$
\begin{gathered}
\leq \frac{r(r-1)\left(a_{2}-a_{1}\right)^{2}}{8 \sqrt[p]{2 p+1}} \\
\times\left\{\sqrt[q]{A\left(\left|a_{1}\right|^{q(r-2)},\left|\frac{a_{1}+a_{2}}{2}\right|^{q(r-2)}\right)}\right. \\
\left.+\sqrt[q]{A\left(\left|\frac{a_{1}+a_{2}}{2}\right|^{q(r-2)},\left|a_{2}\right|^{q(r-2)}\right)}\right\} .
\end{gathered}
$$

Proof. Applying Theorem 2 for $x=\frac{a_{1}+a_{2}}{2}, m=$ $1, n=0, \eta\left(x, m a_{1}\right)=x-m a_{1}, \eta\left(a_{2}, m x\right)=$ $a_{2}-m x, f(x)=x^{r}$ and $\varphi(t)=t$, one can obtain the result immediately.

Proposition 2. Let $a_{1}, a_{2} \in \mathbb{R} \backslash\{0\}$, where $a_{1}<a_{2}$ and $x \in\left[a_{1}, a_{2}\right]$. Then, for $q>1$ and $p^{-1}+q^{-1}=1$, the following inequality hold:

$$
\begin{gathered}
\mid\left(\frac{a_{1}-a_{2}}{2}\right)\left[\frac{1}{H\left(a_{1}^{2}, a_{2}^{2}\right)}+\frac{1}{A^{2}\left(a_{1}, a_{2}\right)}\right] \\
-\left[\frac{1}{H\left(a_{1}, a_{2}\right)}+\frac{1}{A\left(a_{1}, a_{2}\right)}\right]-\frac{2}{L\left(a_{1}, a_{2}\right)} \mid \\
\times\left\{\frac{\leq \frac{\left(a_{2}-a_{1}\right)^{2}}{4 \sqrt[p]{2 p+1}}}{\left.\sqrt[q]{H\left(\left|a_{1}\right|^{3 q},\left|\frac{a_{1}+a_{2}}{2}\right|^{3 q}\right.}\right)}\right. \\
+\frac{\sqrt[q]{H\left(\left|\frac{a_{1}+a_{2}}{2}\right|^{3 q},\left|a_{2}\right|^{3 q}\right)}}{\sqrt{H\left(\mid y^{3 q}\right.}} .
\end{gathered}
$$

Proof. Applying Theorem 2 for $x=\frac{a_{1}+a_{2}}{2}, m=$ $1, n=0, \eta\left(x, m a_{1}\right)=x-m a_{1}, \eta\left(a_{2}, m x\right)=$ $a_{2}-m x, f(x)=\frac{1}{x}$ and $\varphi(t)=t$, one can obtain the result immediately.

Proposition 3. Let $a_{1}, a_{2} \in \mathbb{R} \backslash\{0\}$, where $a_{1}<a_{2}$ and $x \in\left[a_{1}, a_{2}\right]$. Then, for $r \in\{2,3, \ldots\}$ and $q \geq 1$, the following inequality hold:

$$
\begin{gathered}
\mid r\left(\frac{a_{2}-a_{1}}{2}\right)\left[A\left(a_{1}^{r-1}, a_{2}^{r-1}\right)+A^{r-1}\left(a_{1}, a_{2}\right)\right] \\
-\left[A\left(a_{1}^{r}, a_{2}^{r}\right)+A^{r}\left(a_{1}, a_{2}\right)\right]-2 L_{r}^{r}\left(a_{1}, a_{2}\right) \mid \\
\quad \leq \frac{r(r-1)\left(a_{2}-a_{1}\right)^{2}}{48 \sqrt[q]{2}} \mid q \sqrt[q]{A\left(3\left|a_{1}\right|^{q(r-2)},\left|\frac{a_{1}+a_{2}}{2}\right|^{q(r-2)}\right)} \\
+\sqrt[q]{A\left(3\left|\frac{a_{1}+a_{2}}{2}\right|^{q(r-2)},\left|a_{1}\right|^{q(r-2)}\right)}
\end{gathered}
$$




$$
\begin{aligned}
& +\sqrt[q]{A\left(3\left|a_{2}\right|^{q(r-2)},\left|\frac{a_{1}+a_{2}}{2}\right|^{q(r-2)}\right)} \\
& \left.+\sqrt[q]{A\left(3\left|\frac{a_{1}+a_{2}}{2}\right|^{q(r-2)},\left|a_{2}\right|^{q(r-2)}\right)}\right\}
\end{aligned}
$$

Proof. Applying Theorem 3 for $x=\frac{a_{1}+a_{2}}{2}, m=$ $1, n=0, \eta\left(x, m a_{1}\right)=x-m a_{1}, \eta\left(a_{2}, m x\right)=$ $a_{2}-m x, f(x)=x^{r}$ and $\varphi(t)=t$, one can obtain the result immediately.

Proposition 4. Let $a_{1}, a_{2} \in \mathbb{R} \backslash\{0\}$, where $a_{1}<a_{2}$ and $x \in\left[a_{1}, a_{2}\right]$. Then for $q \geq 1$, the following inequality hold:

$$
\begin{gathered}
\mid\left(\frac{a_{1}-a_{2}}{2}\right)\left[\frac{1}{H\left(a_{1}^{2}, a_{2}^{2}\right)}+\frac{1}{A^{2}\left(a_{1}, a_{2}\right)}\right] \\
-\left[\frac{1}{H\left(a_{1}, a_{2}\right)}+\frac{1}{A\left(a_{1}, a_{2}\right)}\right]-\frac{2}{L\left(a_{1}, a_{2}\right)} \mid \\
\times\left\{\frac{q}{\frac{3}{2} \frac{\left(a_{2}-a_{1}\right)^{2}}{24}}\right. \\
+\frac{\sqrt[q]{H\left(3\left|a_{1}\right|^{3 q},\left|\frac{a_{1}+a_{2}}{2}\right|^{3 q}\right)}}{\sqrt{H\left(3\left|\frac{a_{1}+a_{2}}{2}\right|^{3 q},\left|a_{1}\right|^{3 q}\right)}} \\
+\frac{\sqrt[q]{H\left(3\left|\frac{a_{1}+a_{2}}{2}\right|^{3 q},\left|a_{2}\right|^{3 q}\right)}}{\sqrt{H\left(3\left|a_{2}\right|^{3 q},\left|\frac{a_{1}+a_{2}}{2}\right|^{3 q}\right)}} \\
+\frac{\sqrt{H}}{\sqrt{H(1}}
\end{gathered}
$$

Proof. Applying Theorem 3 for $x=\frac{a_{1}+a_{2}}{2}, m=$ $1, n=0, \eta\left(x, m a_{1}\right)=x-m a_{1}, \eta\left(a_{2}, m x\right)=$ $a_{2}-m x, f(x)=\frac{1}{x}$ and $\varphi(t)=t$, one can obtain the result immediately.

Remark 3. Applying our Theorems [2 and 3 for $x=\frac{a_{1}+a_{2}}{2}, m=1, n=0, \eta\left(x, m a_{1}\right)=$ $x-m a_{1}, \eta\left(a_{2}, m x\right)=a_{2}-m x$ and appropriate choices of function $\varphi(t)=\frac{t^{\alpha}}{\Gamma(\alpha)}, \frac{t^{\frac{\alpha}{k}}}{k \Gamma_{k}(\alpha)}, \varphi(t)=$ $t\left(a_{2}-t\right)^{\alpha-1}$, where $f(x)$ is symmetric to $x=$ $\frac{a_{1}+a_{2}}{2}, \varphi(t)=\frac{t}{\alpha} \exp \left[\left(-\frac{1-\alpha}{\alpha}\right) t\right]$, for $\alpha \in(0,1)$, such that $\left|f^{\prime \prime}\right|^{q}$ to be preinvex, we can deduce some new general fractional integral inequalities using above special means. We omit their proofs and the details are left to the interested readers.

\section{Conclusion}

It is expected that from the results obtained, and following the methodology applied, additional special functions may also be evaluated. Future works can be developed in the area of numerical analysis using the theorems and corollaries presented. The authors hope that the ideas and techniques of this paper will inspire interested readers working in this fascinating field.

\section{Acknowledgments}

The authors would like to thank the referee for valuable comments and suggestions.

\section{References}

[1] Aslani, S.M. Delavar, M.R. and Vaezpour, S.M. (2018). Inequalities of Fejér type related to generalized convex functions with applications. Int. J. Anal. Appl., 16(1), 38-49.

[2] Chen, F.X. and Wu, S.H. (2016). Several complementary inequalities to inequalities of Hermite-Hadamard type for $s$-convex functions. J. Nonlinear Sci. Appl., 9(2), 705-716.

[3] Chu, Y.M. Khan, M.A. Khan, T.U. and Ali, T. (2016). Generalizations of HermiteHadamard type inequalities for $M T$-convex functions. J. Nonlinear Sci. Appl., 9(5), 43054316.

[4] Dahmani, Z. (2010). On Minkowski and Hermite-Hadamard integral inequalities via fractional integration. Ann. Funct. Anal., 1(1), 51-58.

[5] Delavar, M.R. and Dragomir, S.S. (2017). On $\eta$-convexity. Math. Inequal. Appl., 20, 203216.

[6] Delavar, M.R. and De La Sen, M. (2016). Some generalizations of Hermite-Hadamard type inequalities. SpringerPlus, 5(1661).

[7] Dragomir, S.S. and Agarwal, R.P. (1998). Two inequalities for differentiable mappings and applications to special means of real numbers and trapezoidal formula. Appl. Math. Lett., 11(5), 91-95.

[8] Khan, M.A. Chu, Y.M. Kashuri, A. Liko, R. and Ali, G. (2018). New Hermite-Hadamard inequalities for conformable fractional integrals. J. Funct. Spaces, Article ID 6928130, 9.

[9] Khan, M.A. Khurshid, Y. and Ali, T. (2017). Hermite-Hadamard inequality for fractional integrals via $\eta$-convex functions. Acta Math. Univ. Comenianae, 79(1), 153-164.

[10] Liu, W.J. (2014). Some Simpson type inequalities for $h$-convex and $(\alpha, m)$-convex 
functions. J. Comput. Anal. Appl., 16(5), 1005-1012.

[11] Liu, W. Wen, W. and Park, J. (2016). Hermite-Hadamard type inequalities for $M T$-convex functions via classical integrals and fractional integrals. J. Nonlinear Sci. Appl., 9, 766-777.

[12] Mihai, M.V. (2013). Some HermiteHadamard type inequalities via RiemannLiouville fractional calculus. Tamkang $J$. Math, 44(4), 411-416.

[13] Mubeen, S. and Habibullah, G.M. (2012). kFractional integrals and applications. Int. $J$. Contemp. Math. Sci., 7, 89-94.

[14] Noor, M.A. Noor, K.I. Awan, M.U. and Khan, S. (2014). Hermite-Hadamard inequalities for $s$-Godunova-Levin preinvex functions. J. Adv. Math. Stud., 7(2), 12-19.

[15] Omotoyinbo, O. and Mogbodemu, A. (2014). Some new Hermite-Hadamard integral inequalities for convex functions. Int. J. Sci. Innovation Tech., 1(1), 1-12.

[16] Özdemir, M.E. Dragomir, S.S. and Yildiz, C. (2013). The Hadamard's inequality for convex function via fractional integrals. Acta Mathematica Scientia, 33(5), 153-164.

[17] Sarikaya, M.Z. and Ertuğral, F. (2020). On the generalized Hermite-Hadamard inequalities. Annals of the University of CraiovaMathematics and Computer Science Series, in press.

[18] Sarikaya, M.Z. and Yildirim, H. (2007). On generalization of the Riesz potential. Indian Jour. of Math. and Mathematical Sci., 3(2), 231-235.

[19] Set, E. Noor, M.A. Awan, M.U. and Gözpinar, A. (2017). Generalized HermiteHadamard type inequalities involving fractional integral operators. J. Inequal. Appl., 169, 1-10.
[20] Wang, H. Du, T.S. and Zhang, Y. (2017). $k^{-}$ fractional integral trapezium-like inequalities through $(h, m)$-convex and $(\alpha, m)$-convex mappings. J. Inequal. Appl., 2017(311), 20.

[21] Weir, T. and Mond, B. (1988). Preinvex functions in multiple objective optimization. $J$. Math. Anal. Appl., 136, 29-38.

[22] Zhang, X.M. Chu, Y.M. and Zhang, X.H. (2010). The Hermite-Hadamard type inequality of $G A$-convex functions and its applications. J. Inequal. Appl., Article ID 507560, 11.

[23] Zhang, Y. Du, T.S. Wang, H. Shen, Y.J. and Kashuri, A. (2018). Extensions of different type parameterized inequalities for generalized $(m, h)$-preinvex mappings via $k$-fractional integrals. J. Inequal. Appl., 2018(49), 30.

Artion Kashuri received his PhD degree from University Ismail Qemali of Vlora in 2016 in the area of Analysis. His research areas are Mathematical Inequalities, Applied Mathematics, Fractional Calculus, Quantum Calculus, etc. He has vast experience of teaching such as Differential Equations, Numerical Analysis, Calculus, Real Analysis, Complex Analysis, Topology, etc. He has more than 100 published papers in international reputation indexed journals. His current position is Lecturer in University Ismail Qemali, Department of Mathematics.

\section{(D) http://orcid.org/0000-0003-0115-3079}

Rozana Liko received her PhD degree from University Ismail Qemali of Vlora in 2018 in the area of Applied Mathematics. Her research areas are Mathematical Inequalities, Applied Mathematics, etc. She has vast experience of teaching such as Probability and Statistics, Calculus, Linear Algebra, Real Analysis, etc. She has more than 50 published papers in international reputation indexed journals. Her current position is Lecturer in University Ismail Qemali, Department of Mathematics.

(1) http://orcid.org/0000-0003-2439-8538

An International Journal of Optimization and Control: Theories \& Applications (http://ijocta.balikesir.edu.tr)

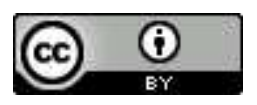

This work is licensed under a Creative Commons Attribution 4.0 International License. The authors retain ownership of the copyright for their article, but they allow anyone to download, reuse, reprint, modify, distribute, and/or copy articles in IJOCTA, so long as the original authors and source are credited. To see the complete license contents, please visit http://creativecommons.org/licenses/by/4.0/. 